\title{
habent sua fata data: der Beitrag der Schweizerischen Akademie der Geistes- und Sozialwissenschaften zur nachhaltigen Sicherung von Forschungsdaten
}

\begin{abstract}
Der Umgang mit digitalen Forschungsdaten gehört heutzutage zum wissenschaftlichen Alltag. Es braucht jedoch geeignete Infrastrukturen, welche die von den Forschenden benötigten Dienstleistungen wie die nachhaltige Datensicherung und -präsentation gewährleisten können. Die Schweizerische Akademie der Geistes- und Sozialwissenschaften, mit einer Fördersumme von jährlich ca. CHF 11,8 Mio. in der Finanzperiode 2017 bis 2020 selbst eine wichtige Datenproduzentin, unterhält deshalb in Zusammenarbeit mit der Universität Basel und weiteren lokalen Satelliten an Schweizer Universitäten das Data and Service Center for the Humanities (DaSCH), welches den Forschenden in den betreffenden Disziplinen zur Verfügung steht. Darüber hinaus setzt sich die Akademie für die Belange von Open Access und Open Data ein. Für die durch die Akademie verantworteten Forschungsinfrastrukturen werden die FAIR Data-Principles verfolgt.
\end{abstract}

\section{Einleitung}

Auch Daten haben Schicksale und Konjunkturen - gegenwärtig stehen diese im Hoch, eine Kehrtwende ist nicht in Sicht. Daten werden überall in den Wissenschaften produziert, auch in den Geistes- und Sozialwissenschaften, datenbasierte Forschungen sind an der Tagesordnung. Die Möglichkeiten, sich die Methoden der Digital Humanities bereits im Studium anzueignen, steigen kontinuierlich, mittlerweile gibt es auch entsprechende Doktorandenschulen. ${ }^{1}$ Nicht die Tatsache, dass Daten nun als Grundlagen für historische, germanistische oder kulturwissenschaftliche Forschungen verwendet werden, ist neu. Die Anfänge der computer-

1 Vgl. hierzu die Zusammenstellungen bei infoclio.ch am 28.12.2017: http://www.infoclio.ch/de/ node/130353 und http://www.infoclio.ch/de/node/136787. Alle Links in diesem Beitrag wurden am 26.03.2018 überprüft.

Anmerkung: ORCID: http://orcid.org/0000-0002-3465-1798

Ә Open Access. (c) Beat Immenhauser, publiziert von De Gruyter. (c) BY-NC-ND Dieses Werk ist lizenziert unter der Creative Commons Attribution-NonCommercial-NoDerivatives 4.0 Lizenz. https://doi.org/10.1515/9783110553796-016 
gestützten geistes- und sozialwissenschaftlichen Studien - etwa quantitative Methoden in der Geschichtswissenschaft oder frühe digitale Editionen - reichen in das letzte Jahrhundert zurück. ${ }^{2}$ Gewandelt hat sich hingegen der Umgang mit den Daten: Heutzutage beginnt sich die Vorstellung durchzusetzen, dass Daten Teil der Forschungsergebnisse sind und deshalb mitveröffentlicht werden sollen, damit jene im Sinne der Transparenz verifizierbar sind. Unter dem Stichwort ,Data Access and Research Transparency“ (DART) werden deshalb Massnahmen zusammengefasst, welche die Nachvollziehbarkeit von Forschungsergebnissen gewährleisten. ${ }^{3}$ Dies gilt etwa für Befragungsdaten als Grundlage sozialwissenschaftlicher Artikel in Periodika, für korpuslinguistische Untersuchungen, prosopographisch angelegte Datenbanken oder digitale Editionen.

Kurzum: Daten allenthalben - damit diese nutzbar sind, bedarf es einer strukturierten, nutzungsoffenen Aggregation in einer nachhaltigen, zeitgemässen Präsentationsform. Ein solcher Zusammenschluss von geistes- oder sozialwissenschaftlichen Daten entspricht in seiner Funktion und Bedeutung etwa einem Datenzentrum für Astrophysik, einer Schweizer Biobanking Platform oder den Datenbanken der Beobachtungsprogramme für Gletscher und Permafrost. ${ }^{4}$ Die Rede ist also von Forschungsinfrastrukturen. Was die Geistes- und Sozialwissenschaften anbelangt, so hat sich in den letzten Jahren ein gemeinsames Verständnis herausgebildet, was darunter zu verstehen ist: Unter Forschungsinfrastrukturen werden Einrichtungen und Dienstleistungen verstanden, die strukturierte, nach transparenten und methodisch nachvollziehbaren wissenschaftlichen Kriterien erarbeitete Sammlungen von Informationen und Wissen für die Ermöglichung von Forschung zur Verfügung stellen. Diese Informationssammlungen sind nachhaltig sowie nutzungsoffen konzipiert und allen interessierten Kreisen zugänglich. ${ }^{5}$ Diese Formel ist so offen konzipiert, dass ganz verschiedene Sammlungen von Informationen und Wissen darin Platz finden. Allen gemeinsam ist

2 Vgl. dazu die Überblicksdarstellung von Manfred Thaller: Geschichte der Digital Humanities. In: Fotis Jannidis, Hubertus Kohle, Malte Rehbein (Hrsg.): Digital Humanities. Eine Einführung. Stuttgart 2017, S. 3-12.

3 Zur DART-Initiative von FORS siehe http://forscenter.ch/en/dart/.

4 Beispiele aus der Schweizer Roadmap für Forschungsinfrastrukturen im Hinblick auf die BFIBotschaft 2017-2020 (2015). https://www.sbfi.admin.ch/dam/sbfi/de/dokumente/schweizer_roa dmapfuerforschungsinfrastrukturenimhinblickaufdiebf.pdf.download.pdf/schweizer_roadmapf uerforschungsinfrastrukturenimhinblickaufdiebf.pdf (Download 28.06.2017).

5 Vgl. SNF - internes Positionspapier: Förderung von Infrastrukturen durch den SNF von Angelika Kalt (05. Juni 2013); vgl. dazu auch die Definition des European Strategy Forum on Research Infrastructures ESFRI: http://ec.europa.eu/research/infrastructures/index_en.cfm?pg=about (Download 28.6.2017). 
das Element der forschungsbasierten Entstehung. ${ }^{6}$ Diese Offenheit kommt auch im entsprechenden Artikel des Forschungsgesetzes, der die Zuständigkeit der Akademien für Forschungsinfrastrukturen regelt, zum Ausdruck: „Sie [die Akademien, B.I.] können Datensammlungen, Dokumentationssysteme, wissenschaftliche Zeitschriften, Editionen oder ähnliche Einrichtungen unterstützen, die als Forschungsinfrastrukturen der Entwicklung von Fachgebieten in der Schweiz dienen und die nicht in die Förderzuständigkeit des SNF oder der Hochschulforschungsstätten fallen oder direkt vom Bund unterstützt werden.“7

\section{Forschungsinfrastrukturenförderung der SAGW}

Die Schweizerische Akademie der Geistes- und Sozialwissenschaften (SAGW) fördert seit den 90er Jahren des 20. Jahrhunderts dauerhafte Einrichtungen, die Grundlagendaten für die Geistes- und Sozialwissenschaften erarbeiten und der Forschung sowie der interessierten Öffentlichkeit zur Verfügung stellen. ${ }^{8}$ Heutzutage betreut die Akademie sieben langfristige Forschungsinfrastrukturen mit jährlich ca. CHF 10,3 Mio. in der Finanzperiode 2017-2020. Dazu kommen noch zehn Herausgeberkommissionen mit jährlichen Administrativbeiträgen von insgesamt CHF 140'000 sowie rund 80 Zeitschriften und Reihen, die ebenfalls als Teil der Infrastrukturförderung betrachtet werden können, mit jährlichen Beiträgen von ca. CHF 1,3 Mio. Gemäss der Leistungsvereinbarung mit dem Staatssekretariat für Bildung, Forschung und Innovation (SBFI) subventioniert die SAGW in der Finanzperiode 2017-2020 geistes- und sozialwissenschaftliche Forschungsinfrastrukturen mit Beiträgen in Höhe von CHF 47,2 Mio. (vgl. Abb. 1). ${ }^{9}$

6 Dazu Markus Zürcher: The Importance of policy-making in setting up and maintaining research infrastructures. In: Brian Kleiner, Isabelle Renschler, Boris Wernli, Peter Farago, Dominique Joye (Hrsg.): Understanding Research Infrastructures in the Social Sciences. Zürich 2013, S. 187-193, hier S. 190.

7 Bundesgesetz über die Förderung der Forschung und der Innovation (FIFG) vom 14. Dezember 2012, Art. 11 Abs. 6 (https://www.admin.ch/opc/de/classified-compilation/20091419/index.html).

8 Ein kurzer Überblick zur Entwicklung der Forschungsinfrastrukturenförderung der SAGW bei Beat Immenhauser: Digital Humanities - ein Thema der SAGW. In: Bulletin der Schweizerischen Akademie der Geistes- und Sozialwissenschaften 4/2015, S. 38f. (http://www.sagw.ch/de/dms/ sagw/bulletins_sagw/bulletins_2015/Bulletin_4_15/Dossier-SAGW_Bulletin_4_15.pdf).

9 Dazu Beat Immenhauser: Forschungsinfrastrukturförderung der Schweizerischen Akademie der Geistes- und Sozialwissenschaften. Swiss Academies Factsheets 2017, vol. 12, no.1. Zenodo: ht tps://doi.org/10.5281/zenodo.802093. 

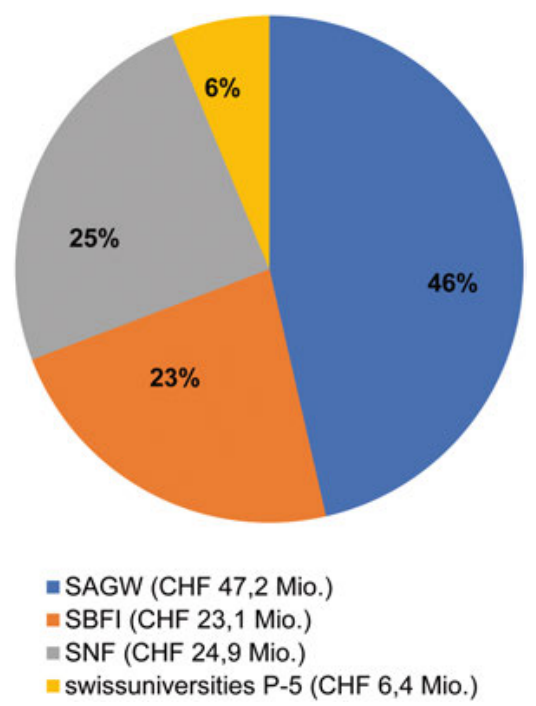

Abb. 1: Subventionen für geisteswissenschaftliche Forschungsinfrastrukturen in der Schweiz (Quelle des Diagramms: https://doi.org/10.5281/zenodo.802093)

Die SAGW gehört zu den wichtigsten Förderorganisationen für geisteswissenschaftliche Forschungsinfrastrukturen in der Schweiz. Der Schweizerische Nationalfonds (SNF), das Staatssekretariat für Bildung Forschung und Innovation sowie swissuniversities über das Förderprogramm P-5 „Wissenschaftliche Information“ unterstützen ebenfalls solche Forschungsinfrastrukturen mit Bundesmitteln. Das Diagramm führt die geplanten Subventionen für diese Projekte für die Periode 2017 bis 2020, mit insgesamt CHF 101,6 Mio., auf. ${ }^{10}$

Der Schweizerische Nationalfonds und die SAGW teilen die Zuständigkeiten für die Förderung von Forschungsinfrastrukturen künftig auf: Der SNF verantwortet weiterhin Editionsprojekte mit einer Laufdauer von nicht mehr als zehn Jahren sowie einzelne weitere geisteswissenschaftliche Forschungsinfrastrukturprojekte. Deutlich länger dauernde Editionen werden nach Ablauf der ersten zehn Förder-

10 Quellenangaben im Einzelnen:

- SAGW: Mehrjahresplanung 2017-2020 (http://www.sagw.ch/dms/sagw/info_sagw/mjp/ Mehrjahresplanung_2017-2020_SAGW_web)

- SBFI: SBFI News 2/2017, S. 10 und 11

- SNF Editionsprojekte: http://www.snf.ch/de/foerderung/infrastrukturen/editionen/Seite n/default.aspx\#Details und http://p3.snf.ch/project-170402

- swissuniversities P-5: https://www.swissuniversities.ch/en/organisation/projects-and-pr ogrammes/p-5/laufende-projekte/. 
jahre von der SAGW zur Weiterführung bis zum Projektende übernommen. In der Periode 2017-2020 werden voraussichtlich acht solcher Editionen zur SAGW transferiert. Ab 2021 figurieren diese Projekte dann in der Mehrjahresplanung der Akademie. Die wissenschaftliche Evaluation dieser Projekte - ihre strategische Bedeutung für die nationale und internationale Profilierung eines Fachgebiets wurde bereits im Vorfeld positiv evaluiert - wird durch ein gemeinsames Board von Spezialistinnen und Spezialisten des SNF und der SAGW sichergestellt. Ab 2021 wird die SAGW damit ihr Fördervolumen um weitere ca. CHF 10,4 Mio. ausbauen, so dass sie eine Fördersumme von voraussichtlich CHF 58 Mio. von 2021 bis $2024 \mathrm{zu}$ verwalten haben wird.

Der SAGW kommt damit eine hohe Verantwortung $\mathrm{zu}$, sich aktiv um eine hochstehende Qualität, eine nachhaltige Speicherung sowie um eine zeitgemässe Präsentation der in den Infrastrukturen vorgehaltenen Daten zu kümmern. Im Bereich der Zeitschriftenförderung hat die Akademie bereits im September 2016 eine Open Access-Strategie publiziert, welche die subventionierten Periodika bis 2020 zum Green oder zum Gold Open Access führen soll. Weitere Schwerpunkte der Strategie sind unter anderem Retrodigitalisierungsprojekte der älteren Ausgaben der Periodika, die Einführung von persistenten Identifikatoren für Artikel (DOI) sowie für Autorinnen und Autoren (ORCID) und Angaben zu den Nutzungsrechten (CC-Lizenzen). ${ }^{11}$

Für die laufende Mehrjahresperiode bis 2020 hat es sich die Akademie überdies zum Ziel gesetzt, für ihre langfristigen Forschungsunternehmen eine gemeinsame Data Policy einzuführen. Sie ist damit in guter Gesellschaft: Der SNF hat als eine der ersten nationalen Forschungsförderungsorganisationen Open Research Data-Grundsätze festgelegt, deren Einhaltung Gesuchsstellende in Form eines Datenmanagementplans nachweisen müssen. ${ }^{12}$ Kernstück dieser Grundsätze ist die Vorhaltung der Daten in einem entsprechenden Repositorium. Damit bildet das 2017 offiziell gegründete Data and Service Center for the Humanities (DaSCH) eine wichtige Schnittstelle zwischen den Bedürfnissen der Fachgemeinschaft und den Policies der Förderorganisationen.

11 Zur SAGW-Strategie: http://www.sagw.ch/sagw/laufende-projekte/open-access/oa-strategie. html; englische Version auf zenodo: https://doi.org/10.5281/zenodo.189093.

12 Dazu http://www.snf.ch/de/derSnf/forschungspolitische_positionen/open_research_data. Der österreichische Wissenschaftsfonds hat ein Pilotprogramm Open Research Data mit zwölf Projekten gestartet: https://www.fwf.ac.at/de/news-presse/news/nachricht/nid/20170313-2232/. 


\section{Das Data and Service Center for the Humanities (DaSCH)}

Vor zehn Jahren, im November 2007, verabschiedeten 210 Teilnehmerinnen und Teilnehmer auf der SAGW-Tagung Das digitale Gedächtnis der Schweiz: Stand, Herausforderungen und Lösungswege das Memorandum Empfehlungen zur Sicherung des nationalen Kulturgutes der Schweiz im digitalen Zeitalter zuhanden des Eidgenössischen Departements des Innern. Gefordert wurden damals:

1. langfristige Sicherstellung der technischen Benutzbarkeit von Daten

2. Regulierung im Bereich der Sicherung und der Kooperation

3. laufende Evaluation der bestehenden Infrastrukturen und Identifikation des Entwicklungsbedarfs in Zusammenarbeit mit Dritten (Universitäten und private Firmen)

4. Koordinationsfunktionen im technischen und inhaltlichen Bereich sowie in der Ausbildung ${ }^{13}$

Am 01. Januar 2017 konnte das DaSCH nach einer zehnjährigen Vorbereitungsphase seinen regulären Betrieb aufnehmen (http://www.dasch.swiss/). Damit besteht nun für die Geisteswissenschaften ein Angebot zum Punkt 1 der damaligen Forderungen. Die übrigen Punkte sind in Bearbeitung oder realisiert, auch wenn eine eigentliche Regulierung im Bereich der Sicherung und Kooperation heutzutage nicht mehr angestrebt wird, da man sich eher an Standards orientiert. Die Evaluationen der langfristigen Editionsprojekte verantwortet seit 2016 ein gemeinsames Board des SNF und der SAGW. ${ }^{14}$ Mit unterstützenden Koordinationsaufgaben für die Sicherung von geisteswissenschaftlichen Forschungsdaten ist die SAGW überdies gemäss der Botschaft für Bildung, Forschung und Innovation 2017-2020 explizit beauftragt worden. ${ }^{15}$

So weit - so gut: Wir haben nun ein Datenzentrum, das den spezifischen Anforderungen der Geisteswissenschaften entspricht. Diese zeichnen die folgenden Spezifika aus:

- Geisteswissenschaftliche Forschungsdaten sind sehr vielfältig. Das gesamte Medienspektrum von audiovisuellen Daten und Texten in unterschiedlichen

13 Zum Memorandum siehe http://www.sagw.ch/de/sagw/oeffentlichkeitsarbeit/stellungnah men/sn-2007/sn-memopolitik.html.

14 Siehe z.B. Leistungsvereinbarung zwischen Bund und SAGW 2017 bis 2020, S. 21 (zenodo: htt ps://doi.org/10.5281/zenodo.822393).

15 Siehe BFI-Botschaft 2017-2020, S. 3195 (https://www.sbfi.admin.ch/dam/sbfi/de/dokumen te/2016/cst/bfi_17-20.pdf.download.pdf/BFI-17-20_d.pdf). 
Organisationsformen wie Datenbanken, Sammlungen, Editionen oder Webseiten in verschiedenen Formaten muss berücksichtigt werden können, da Vorgaben, etwa welche Software und welche Formate akzeptiert werden, seitens der Forschenden als Bevormundung wahrgenommen werden können.

- Die im DaSCH gespeicherten Daten müssen über eine längere Zeit, mehrere Jahrzehnte, verfügbar sein. Einmal abgeschlossene, nach modernen Kriterien erarbeitete digitale Editionen werden in aller Regel nicht nach zehn Jahren wieder neu herausgegeben, so dass die Daten wie früher Editionen in Buchform über mehrere Generationen von Forschenden hinweg nutzbar sein müssen.

- Geisteswissenschaftliche Wissenschaftstraditionen bringen es mit sich, dass eine hohe Zitierfähigkeit der vorgehaltenen Daten gewährleistet werden muss, und zwar in diesem Zustand, wie eine Forscherin oder ein Forscher jene vorgefunden haben. Deshalb muss DaSCH eine Versionsverwaltung anbieten können. Die Zitierfähigkeit muss zudem permanent erhalten bleiben.

- Ein auch in den Geisteswissenschaften notwendiges Erfordernis betrifft den abgestuften Zugang zu den Daten. Auch wenn die Daten grundsätzlich nach der Maxime ,open by default‘ angeboten werden sollen, muss es dennoch möglich sein, Daten ganz oder teilweise zumindest temporär einem definierten Nutzerkreis vorzubehalten.

- Schliesslich ist die Wiederverwendbarkeit der Daten ganz zentral und muss unter allen Umständen permanent gewährleistet werden können.

Die technischen und organisatorischen Lösungen für die genannten Herausforderungen wurden in einem Pilotprojekt von 2013 bis 2016 erarbeitet. ${ }^{16}$ Die Plattform besteht aus den Hauptkomponenten KNORA (Knowledge Organization, Representation and Annotation) und SALSAH (System for Annotation and Linkage of Sources in Arts and Humanities). KNORA ist ein Repositorium, basierend auf semantischen Webtechnologien (RDF, RDFS und OWL) und bietet eine RESTful Schnittstelle für den Zugang. SALASH ist ein generisches, webbasiertes Frontend, das Tools für eine virtuelle Forschungsumgebung zur Verfügung stellt. Die Codes sind Open Source unter einer GNU-Lizenz zugänglich. ${ }^{17}$

16 Das Pilotprojekt ist detailliert dokumentiert im Schlussbericht: Schweizerische Akademie der Geistes- und Sozialwissenschaften (2015), Final report for the pilot project „Data and Service Center for the Humanities“ (DaSCH), Swiss Academies Reports 10 (1) (https://doi.org/10.5281/ zenodo.822918). Weitere Informationen zum DaSCH sind auf der Webseite der SAGW zu finden: http://www.sagw.ch/dasch.

17 https://github.com/dhlab-basel/Knora; siehe zudem das Factsheet zum DaSCH: http://www. sagw.ch/de/dms/sagw/unternehmen/dasch/DaSCH-17-3_Factsheet/Factsheet.pdf. 
Alle Daten in der KNORA-Plattform sind nach dem Datenmodell des Resource Description Framework (RDF) organisiert. Dieses Modell wurde ursprünglich für den Datenaustausch im semantischen Web entwickelt und wird von einer grossen internationalen Community weiterentwickelt. ${ }^{18}$ Dies bedeutet, dass die Daten eines Projekts, die in die Plattform des DaSCH integriert werden sollen, zunächst in das RDF-Modell umgewandelt werden müssen, sofern das Projekt nicht bereits ,ab ovo' im DaSCH erstellt worden ist. Für das DaSCH-Projektteam bedeutet die Datenübernahme je nach Zustand und Komplexität des Projekts jeweils einen gewissen Aufwand, der aber mittelfristig abnehmen wird, da Elemente einmal entwickelter Schnittstellen zwischen Projekten und dem DaSCH wiederverwendet werden können und weil künftig die Daten neuer Projekte immer häufiger gleich im RDF-Format angelegt werden dürften. Die zentralen Vorteile dieses Konzepts sind:

1. Aufgrund der flexiblen Möglichkeiten der Plattform können verschiedenste Datenformate (z.B. Text Encoding Initiative (TEI)-Codierungen, wie sie für Editionen relevant sind, oder Filemaker-Datenbanken) in KNORA abgebildet werden. Damit entfällt die sonst bei anderen Datenzentren zu beobachtende Einschränkung auf archivfähige Formate.

2. Aufgrund des festen Sets von verwendeten Datenformaten in der Plattform entfällt die individuelle Migration einzelner Datenbestände in jeweils neuere Versionen, damit die Zugänglichkeit gewährleistet werden kann. Dies erlaubt eine sehr kostengünstige Wartung des gesamten Systems.

3. Die Schnittstellen zwischen den Datenressourcen und den Nutzenden, beispielsweise die Suchmöglichkeiten in Datenbanken, können so nachgebildet werden, dass kaum ein Unterschied zwischen der vorherigen und der Version im DaSCH festgestellt werden kann - ausser, dass die Nutzenden zusätzliche Werkzeuge wie ein Annotationstool oder persistente Identifikatoren zur Verfügung haben.

Damit unterscheidet sich die KNORA-Plattform von jenen Datenrepositorien, die auf Selbstarchivierung der Daten durch die Forschenden beruhen. ${ }^{19}$ Da mit dem

18 Weitere Informationen unter: https://www.w3.org/RDF/.

19 Beispielsweise das Repositorium zenodo, das durch das CERN und das OpenAIRE-Konsortium verwaltet wird (https://zenodo.org/). Die Datenplattform Huma-Num, eine Très grande infrastructure de recherche (TGIR) des humanités numériques in Frankreich beruht hingegen auf der gleichen Technologie wie das DaSCH (http://www.huma-num.fr/la-tgir-en-bref). Vgl. zum DaSCH zudem: Lukas Rosenthaler, Beat Immenhauser, Peter Fornaro: Implementation of a National Data Center for the Humanities (DaSCH). In: Digital Humanities 2016: Conference Abstracts. Jagielloni- 
DaSCH ein umfassendes Beratungsangebot für die geisteswissenschaftliche Fachgemeinschaft zu Standards und IT- beziehungsweise Digital Humanities-Technologien für die Sicherung, Pflege und Weiterverwendung von Daten verbunden ist, wird mit allen Projektverantwortlichen zunächst abgeklärt, wie das Projekt auf der Plattform präsentiert werden soll. In einem weiteren Schritt wird dann das Übernahmeprozedere festgelegt. ${ }^{20}$

Die strategische Führung des Datenzentrums nimmt eine durch die SAGW eingesetzte Kommission wahr, in der die relevanten Stakeholder vertreten sind und damit eine breite Abstützung und Verankerung des DaSCH in der Schweiz und international sicherstellen. ${ }^{21}$ Indem die Akademie das DaSCH in Partnerschaft mit der Universität Basel als nationales Langzeitunternehmen analog zu anderen Forschungseinrichtungen der Akademie betreibt, können einerseits die Anbindung an die universitäre Forschung und andererseits die Nachhaltigkeit optimal gewährleistet werden.

\section{Herausforderungen für die Zukunft}

Damit sich das DaSCH nachhaltig etablieren kann, bedarf es zweierlei: höherer Zuschüsse und eines Netzwerks von lokalen Satelliten. Die Bundessubventionen von jährlich CHF 500'000 bis 2020 werden nicht ausreichen, um das Datenzentrum in die Lage zu versetzen, den vorhandenen Bedürfnissen nach Beratung, Expertise und Datensicherung zu entsprechen. Die SAGW wird sich für zusätzliche Mittel ab 2021 einsetzen. Aufgrund der Auflage des SNF, dass Gesuchsstellende ab 01. Oktober 2017 Datenmanagementpläne für neue Projekte vorlegen müssen, ist mit einer steigenden Nachfrage nach Informationen, wie eine solche Planung gemacht werden kann, zu rechnen. Es wird an den einzelnen Universitäten beziehungsweise Fakultäten sein, entsprechende Anlaufstellen zu bezeichnen. Das DaSCH wird subsidiäre Dienstleistungen übernehmen. Die Einrichtung von Satelliten für einen first level support an weiteren geisteswissenschaftlichen Fakultäten erhält damit zusätzliche Dringlichkeit. Sollte es gelingen, und es gibt Anzeichen, dass nebst dem Lausanner Satellit ${ }^{22}$ auch noch Einrich-

an University \& Pedagogical University, Kraków, S. 339-341 (https://doi.org/10.5281/zenodo. 821581).

$20 \mathrm{Zu}$ den Dienstleistungen des DaSCH vgl. http://www.sagw.ch/de/dms/sagw/unternehmen/d asch/DaSCH-17-7_Gescha-ftsprozesse/Customer\%20Relationship-Prozesse.pdf.

21 Zum Mandat der Kommission und zu deren Zusammensetzung siehe http://www.sagw.ch/da sch.

22 Zum Lausanner Satellit siehe https://platec.unil.ch/. 
tungen in Zürich, Bern und Luzern demnächst folgen werden, kann auf diese Weise die nationale Positionierung des DaSCH gefördert werden.

Darüber hinaus stehen wir noch vor einer ganzen Reihe weiterer Herausforderungen, was die geistes-und sozialwissenschaftlichen Forschungsinfrastrukturen in der Schweiz anbelangt. Zwei Punkte seien an dieser Stelle erwähnt:

1. Förderung von geistes- und sozialwissenschaftlichen Forschungsinfrastrukturen

Die vom SBFI seit mehreren Jahren verfolgte Strategie, primär die Hochschulen mit der Finanzierung und Führung von Forschungsinfrastrukturen, mit Ausnahme besonders kostenintensiver Einrichtungen, in die Pflicht $\mathrm{zu}$ nehmen, hat bisher aus unserer Perspektive nicht zur Klärung der Zuständigkeit geführt. ${ }^{23}$ Nachdem immerhin die Zuständigkeitsfrage für Editionsprojekte gelöst werden konnte, bedarf es nun noch gemeinsamer Anstrengungen, um eine vergleichbare Lösung für die weiteren geistes- und sozialwissenschaftlichen Forschungsinfrastrukturen zu finden. Damit zusammen hängt das Desiderat einer Förderlinie für kleinere oder mittelgrosse Forschungsinfrastrukturprojekte in den Geistes- und Sozialwissenschaften - Projekte, die beispielsweise Digitalisierungen, technologische Weiterentwicklungen oder Valorisierungen bestehender Angebote zum Ziel haben. Ein solches Angebot wird dringend nötig sein, wenn man unter Digitalisierung nicht nur die Realisierung von Industrie 4.0, sondern auch die Grundlagen für Digital Humanities und Open Science in den Geistes- und Sozialwissenschaften schaffen will. ${ }^{24}$

\section{SAGW und Open Data}

Auch in der direkten Zuständigkeit der SAGW sind Herausforderungen anzugehen: Die Akademie ist gewillt, die ihr zugeordneten Forschungsinfrastrukturen fit für die Zukunft zu machen und sie insbesondere in Richtung Open Science weiter zu entwickeln. Dies bedeutet im Einzelnen:

23 Siehe beispielsweise Schweizer Roadmap für Forschungsinfrastrukturen 2019 (im Hinblick auf die BFI-Planung 2021-2024). Zielsetzung, Prozess und Kriterien: ein Leitfaden (SBFI 2017), S. 3 (https://www.sbfi.admin.ch/dam/sbfi/de/dokumente/2017/03/ch-roadmap-2019.pdf.download. pdf/170308_Leitfaden_Roadmap_2019_SBFI_DE.pdf).

$24 \mathrm{Zu}$ diesem Schluss kommt zumindest implizit auch der vom Eidgenössischen Departement für Wirtschaft, Bildung und Forschung (WBF) und dem SBFI erarbeitete Bericht Herausforderungen der Digitalisierung für Bildung und Forschung in der Schweiz vom Juli 2017, S. 66 (https://www.sbfi. admin.ch/dam/sbfi/de/dokumente/2017/06/bericht-digitalisierung.pdf.download.pdf/bericht_di gitalisierung_d.pdf). 
- Alle Forschungsinfrastrukturen sollen bis 2020 über eine transparente DataPolicy auf der Grundlage der FAIR Data-Principles verfügen. Gemäss diesen Principles sollen Daten auffindbar, frei zugänglich, kompatibel und wieder verwendbar sein. ${ }^{25}$

- Grundsätzlich soll gelten: open by default. Die Standardpräsentation der Daten sollte möglichst offen und frei zugänglich gestaltet sein, ohne Sperrfristen, Abweichungen sind jedoch klar begründet.

- Die Präsentationsform ist auf eine möglichst optimale Wiederverwendung der Daten ausgerichtet. Die Daten verfügen deshalb über suchbare Metadaten, über eindeutige Identifikatoren und über CC-Lizenzen.

- Die nachhaltige Sicherung und Verfügbarkeit der Daten sind gewährleistet.

- Wenn möglich verfügen die einzelnen Forschungsinfrastrukturen über eine Zertifizierung, beispielsweise das Data Seal of Approval ${ }^{26}$.

- Die SAGW bemüht sich im Rahmen der Mehrjahresplanung 2021-2024 um eine kontinuierliche, nachhaltige Finanzierung der Forschungsinfrastrukturen.

- Mit einer fachlichen und administrativen Begleitung der Projekte sowie mit regelmässigen Evaluationen sollen die wissenschaftliche Qualität und ein gutes Management der Forschungsinfrastrukturen gefördert und überprüft werden.

Viele der durch die SAGW betreuten Forschungsinfrastrukturen befinden sich bereits auf gutem Wege - die Akademie ist bestrebt, sie weiterhin darin zu bestärken und zu begleiten, damit deren Daten nicht eines Tages das Schicksal des digitalen Vergessens erleiden.

25 Fair Data-Principles: https://www.force11.org/fairprinciples; vgl. auch Mark D. Wilkinson u. a.: The FAIR Guiding Principles for scientific data management and stewardship. In: SCIENTIFIC DATA 3:160018 (https://dx.doi.org/10.1038/sdata.2016.18).

26 Siehe https://datasealofapproval.org/en/. Das Data Seal of Approval wird 2018 voraussichtlich durch das CoreTrustSeal abgelöst, vgl. https://www.coretrustseal.org/. 